SLAC-PUB-8674

August, 2000

\title{
Estimates of Emittance Dilution and Stability in High-Energy Linear Accelerators*
}

\author{
T.O. Raubenheimer \\ Stanford Linear Accelerator Center, Stanford University, Stanford, CA 94309
}

\begin{abstract}
In this paper, we present a series of analytic expressions to predict the beam dynamics in a long linear accelerator. These expressions can be used to model the linac optics, calculate the magnitude of the wakefields, estimate the emittance dilution due to misaligned accelerator components, and estimate the stability and jitter limitations. The analytic expressions are based on the results of simple physics models and are useful to understand the parameter sensitivities. They are also useful when using simple codes or spreadsheets to optimize a linac system.
\end{abstract}

Submitted to Physical Review Special Topics: Accelerators and Beams

*Work supported by Department of Energy contract DE-AC03-76SF00515. 


\title{
Estimates of Emittance Dilution and Stability in High-Energy Linear Accelerators
}

\author{
T.O. Raubenheimer \\ Stanford Linear Accelerator Center \\ P.O. Box 4349, Stanford, CA 94309
}

(August 16, 2000)

\begin{abstract}
In this paper, we present a series of analytic expressions to predict the beam dynamics in a long linear accelerator. These expressions can be used to model the linac optics, calculate the magnitude of the wakefields, estimate the emittance dilution due to misaligned accelerator components, and estimate the stability and jitter limitations. The analytic expressions are based on the results of simple physics models and are useful to understand the parameter sensitivities. They are also useful when using simple codes or spreadsheets to optimize a linac system.
\end{abstract}

\section{INTRODUCTION}

At the present time a number of high performance linear accelerators are being designed for short wavelength FELs $[1,2]$, future linear colliders [3-7], or as injectors for storage rings [8,9]. In this paper, we will present a number of relatively simple formula to parameterize such systems. In particular, we present formula to model the optics, estimate the transverse and longitudinal wakefields, and estimate the emittance dilution and stability in high-energy linear accelerators. The utility of these relations is to allow rapid calculation of the primary parameters and estimate the performance of such a system. In addition, the analytic results explicitly illustrate the parameter sensitivities and thus can be a useful tool when understanding the optimization of the accelerators.

Although our results are more general, the derivations of the expressions essentially follow those in Refs. [10-12] and, for brevity, will not be presented here. We will however provide extensive references pointing to detailed derivations and extensions of the concepts discussed and modeled. Throughout, we will assume ultra-relativistic particles with $\beta \approx 1$ and will only briefly consider the effect of space charge forces. Our estimates assume random distributions of errors and include the effect of simple trajectory correction. Usually, the calculations are based on simple two particle models and, it should be noted, these expressions are not intended to yield $10 \%$ results - that accuracy must be obtained with detailed computer simulation/calculations. Instead, these expressions typically produce answers that are within a factor of two of those from computer simulation and, more importantly, describe the dependence on the primary parameters. Another recent calculation is described in Ref. [13] which provides more accurate expressions for a limited set of effects and parameters.

In the following, we will present the relevant equations of motion. Then, we describe a parameterization of the longitudinal and transverse wakefields and discuss the BNS damping/autophasing conditions which are used to control the single-bunch beam break-up (bbu). Next, we will present estimates for the primary sources of single- and then multi-bunch emittance dilution. This is followed by a description of the jitter and stability issues. Finally, we discuss the probability distribution for the emittance dilutions and then describe some of the more advanced correction techniques. Throughout the paper, we will illustrate many of the calculations with examples from the Stanford Linear Collider (SLC) [14] linac and the Next Linear Collider (NLC) linac [3].

\section{EQUATIONS OF MOTION}

In a high-energy linear accelerator, the longitudinal beam distribution is essentially frozen. In this case, the energy of a particle $\gamma=E / m c^{2}$ can be written:

$$
\frac{d}{d s} \gamma(s, z)=\frac{e}{m c^{2}} \mathcal{E}_{\mathrm{rf}}(s) \cos \left(z \kappa_{r f}+\phi\right)+4 \pi \epsilon_{0} N r_{e} \int_{z}^{\infty} W_{\|}\left(s, z^{\prime}-z\right) \rho\left(z^{\prime}\right) d z^{\prime},
$$

where $s$ and $z$ are the longitudinal position in the accelerator and in the bunch, $\mathcal{E}_{\text {rf }}$ and $\kappa_{r f}$ are the acceleration gradient and the rf wavenumber, and $\phi$ is the rf phase at the center of the bunch. In addition, $W_{\|}$and $\rho$ are the monopole longitudinal wakefield and the longitudinal beam distribution where $W_{\|}$has units of $V / C / m$. Finally, $N$, $r_{e}$, and $\epsilon_{0}$ are the number of particles per bunch, the classical electron radius, and the permittivity of free space: 
$\epsilon_{0}=8.85 \times 10^{-12} \mathrm{~F} / \mathrm{m}$. Note that in this definition, the bunch is centered at $z=0$ and the head of the bunch is at positive $z$.

Of course, this equation is only correct if the slippage of a particle in the bunch is small compared to the bunch length. The actual slippage of a particle depends on both the energy deviation and on its transverse oscillation amplitude:

$$
\frac{d}{d s} z \approx \frac{J}{2 \beta}-\frac{\Delta \gamma}{\gamma^{3}}
$$

where $\beta$ is the transverse beta function, $\Delta \gamma$ is the energy deviation, and $J$ is the particle action:

$$
J \equiv \frac{1}{2}\left[\frac{1+\alpha^{2}}{\beta} y^{2}+2 \alpha y y^{\prime}+\beta y^{\prime 2}\right]
$$

As stated, both of these contributions are negligible for typical parameters.

Now, the transverse equation of motion can be written:

$$
\begin{aligned}
& \frac{1}{\gamma} \frac{d}{d s} \gamma \frac{d}{d s} y(s, z)-(1-\delta) K\left(y-y_{q}\right)= \\
& \quad(1-\delta) G-4 \pi \epsilon_{0} N r_{e} \frac{1-\delta}{\gamma_{0}} \int_{z}^{\infty} W_{\perp}\left(s, z^{\prime}-z\right)\left(y(z)-y_{a}\right) \rho\left(z^{\prime}\right) d z^{\prime},
\end{aligned}
$$

where $s$ and $z$ are the longitudinal position in the accelerator and the bunch and $\delta$ is the relative energy deviation:

$$
\delta(s, z) \equiv \frac{\gamma-\gamma_{0}}{\gamma}
$$

In addition, $\gamma_{0}$ is the nominal beam energy, $K$ and $G$ are the normalized quadrupole and bending fields, $y_{q}$ and $y_{a}$ are the quadrupole and accelerator structure misalignments, and $W_{\perp}$ is the transverse dipole wakefield in units of $V / C / m^{2}$.

In these expressions, we have ignore the space charge forces due to the mutual repulsion of the particles. At high energy, these forces are usually very small compared to the other effects however, for completeness, we can estimate their magnitude. In the longitudinal, the space charge force should be compared to the variation of the rf acceleration and the wakefields. Assuming a uniform beam, it can be estimated as:

$$
\frac{d}{d s} \gamma_{s c}(s, z) \approx-\frac{2 N r_{e}}{\gamma^{2}} \frac{d}{d z} \rho(z)\left(\ln \frac{b}{a}+\frac{1}{2}\right)
$$

where $b$ and $a$ are the vacuum chamber radius and the beam radius, respectively. Further assuming a Gaussian longitudinal profile, this can be expressed as:

$$
\Delta \gamma_{s c} \approx \frac{N r_{e}}{\sigma_{z}^{2}} \int \frac{d s}{\gamma^{2}}\left(\ln \frac{b}{a}+\frac{1}{2}\right)
$$

where $\Delta \gamma_{s c}$ is the full width of the induced energy spread.

In the transverse, the space charge forces should be compared with the external focusing. This can be parameterized in terms of the depression of the betatron phase advance:

$$
\Delta \nu_{s c x, y} \approx-\frac{N r_{e}}{(2 \pi)^{3 / 2} \sigma_{z}} \int d s \frac{\beta_{x, y}}{\gamma^{3} \sigma_{x, y}\left(\sigma_{x}+\sigma_{y}\right)} .
$$

When considering flat beams $\left(\sigma_{x} \gg \sigma_{y}\right)$ in a FODO lattice where the product $\beta_{x} \beta_{y}$ is roughly constant, this can be re-written in terms of the normalized emittances:

$$
\Delta \nu_{s c x, y} \approx-\frac{N r_{e}}{(2 \pi)^{3 / 2} \sigma_{z}} \frac{1}{\sqrt{\gamma \epsilon_{x, y} \gamma \epsilon_{x}}} \int \frac{d s}{\gamma^{2}}
$$

In both the longitudinal and transverse, the space charge forces tend to be small at high energy. This arises, in the longitudinal plane, because of the Lorentz contraction and, in the transverse plane, because of the cancellation of the electric and magnetic forces in a parallel beam. When the beam is converging or diverging this $1 / \gamma^{2}$ reduction is replaced by a term that depends on the square of the angular divergence and the space charge forces can be more important [15]. 


\section{BEAM EMITTANCE}

Throughout this paper, we will discuss the projected 2-D rms normalized emittance, defined as:

$$
\gamma \epsilon_{y}=\gamma \sqrt{\left(\left\langle y^{2}\right\rangle-\langle y\rangle^{2}\right)\left(\left\langle y^{\prime 2}\right\rangle-\left\langle y^{\prime}\right\rangle^{2}\right)-\left(\left\langle y y^{\prime}\right\rangle-\langle y\rangle\left\langle y^{\prime}\right\rangle\right)^{2}}
$$

where the angle brackets denote an average over the beam particles. Dilutions of this emittance will arise due to changes in the particle positions or angles. Dropping the explicit centroid subtraction and assuming at all averages are calculated about the centroid, the resulting emittance can be written:

$$
\gamma \epsilon_{y}^{2}=\gamma^{2}\left(\left(\sigma_{y}^{2}+\left\langle\Delta y^{2}\right\rangle\right)\left(\sigma_{y^{\prime}}^{2}+\left\langle\Delta y^{\prime 2}\right\rangle\right)-\left(\sigma_{y y^{\prime}}+\left\langle\Delta y \Delta y^{\prime}\right\rangle\right)^{2}\right)
$$

where $\sigma_{y}^{2}=\left\langle y^{2}\right\rangle, \ldots$, and we assumed that there are no correlations between the errors and the individual particle positions and angles within the beam. We will further assume that $\left\langle\Delta y \Delta y^{\prime}\right\rangle^{2}=\left\langle\Delta y^{2}\right\rangle\left\langle\Delta y^{\prime 2}\right\rangle$ which occurs because either the error is correlated in $y$ and $y^{\prime}$ or the error only causes a change in position or angle but not both simultaneously. Both of these assumptions are valid for all of the errors that we will discuss.

In this case, we can express the emittance as:

$$
\gamma \epsilon=\gamma \epsilon_{0} \sqrt{1+2 \Delta \gamma \epsilon / \gamma \epsilon_{0}}
$$

where $\gamma \epsilon_{0}$ is the initial emittance and

$$
\Delta \gamma \epsilon=\frac{\gamma}{2}\left(\frac{1+\alpha_{\star}^{2}}{\beta_{\star}} \Delta y^{2}+2 \alpha_{\star} \Delta y \Delta y^{\prime}+\beta_{\star} \Delta y^{\prime 2}\right)
$$

where $\alpha_{\star}$ and $\beta_{\star}$ are the beam parameters: $\beta_{\star}=\sigma_{y}^{2} / \epsilon$ and $\alpha_{\star}=-\left\langle y y^{\prime}\right\rangle / \epsilon$, which are equal to the lattice parameters $\alpha$ and $\beta$ when the beam is matched to the lattice; the beam and lattice parameters will be discussed further in Section VIII A.

Note that $\Delta \gamma \epsilon$ adds linearly to the projected emittance when the dilutions are small but has a more complicated dependence in general. However, assuming that the beam is initially matched to the lattice, after the emittance is diluted, the beam will be mismatched and thus the emittance dilution will filament. After filamentation, the final rms emittance is given by the linear addition of the initial emittance and the dilution [16]:

$$
\gamma \epsilon=\gamma \epsilon_{0}+\Delta \gamma \epsilon
$$

although the beam distribution will also evolve; filamentation will also be discussed in Section VIII A.

\section{LINAC MODEL}

In the calculations that follow, we will assume that the horizontal and vertical focusing strengths are equal and the focusing structure of the linac scales in a simple manner with the beam energy. In particular, we assume that the linac is constructed from FODO cells whose length increases with beam energy as $L_{\text {cell }}(s) \propto \gamma(s)^{\alpha}$. Furthermore, we assume that the quadrupole focal length scales in the same manner-this keeps the phase advance per cell constant and causes the beta functions to scale with the cell length. Thus,

$$
L_{\text {cell }}(s) \propto \gamma(s)^{\alpha} \quad K L_{\mathrm{q}}(s) \propto \gamma(s)^{-\alpha} \quad \beta(s) \propto \gamma(s)^{\alpha} \quad \psi_{\text {cell }}(s) \propto \text { const } .
$$

This scaling is convenient because it allows straightforward calculation of the optical functions. Assuming thin quadrupoles, which are short compared to the cell length, the integrated quadrupole strength can be expressed in terms of the cell length and the phase advance:

$$
K L_{\mathrm{q}}= \pm \frac{4 \sin \left(\psi_{c} / 2\right)}{L_{\text {cell }}}
$$

and the beta functions at the quadrupoles are:

$$
\beta=L_{\text {cell }} \frac{1 \pm \sin \left(\psi_{\text {cell }} / 2\right)}{\sin \psi_{\text {cell }}} .
$$


In these expressions, the positive signs are for the maximum beta function at the focusing quadrupoles and the negative signs are for the minimum beta function at the defocusing quadrupoles. Furthermore, the average beta function through the cell can be written:

$$
\bar{\beta}=\frac{L_{\text {cell }}}{2}\left(\cot \frac{\psi_{\text {cell }}}{2}+\frac{2}{3} \tan \frac{\psi_{\text {cell }}}{2}\right)
$$

and the average chromaticity per FODO cell is:

$$
\xi=-\frac{\tan \left(\psi_{\text {cell }} / 2\right)}{\pi} .
$$

Finally, using this scaling, the number of FODO cells in the linac can be expressed:

$$
N_{\text {cell }}=\frac{\gamma_{i}}{(1-\alpha) L_{\text {cell } i} G}\left[\left(\frac{\gamma_{f}}{\gamma_{i}}\right)^{1-\alpha}-1\right]
$$

where the subscripts $i$ and $f$ denote initial and final values, $G$ is the loaded gradient in units of $m c^{2}$, and the value at $\alpha=1$ is given by the limiting expression:

$$
\lim _{\alpha=1} \frac{\left(x^{\alpha}-1\right)}{(1-\alpha)}=\ln (x)
$$

In some cases, independent variation of the cell length and focal length has been included in the linac lattice parameterization [17]. This has the effect of causing the phase advance per cell to change as a function of energy, but since this variation is typically small, it can usually be ignored in the dilution estimates.

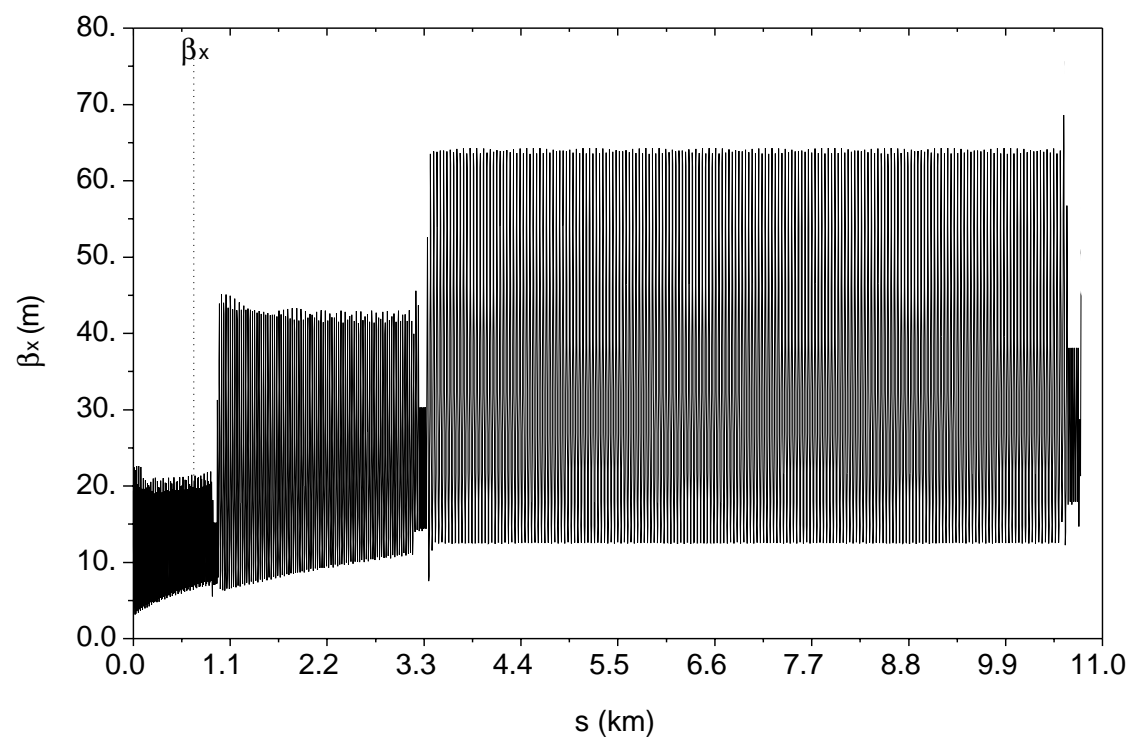

FIG. 1. Horizontal beta-function in the NLC linac.

Finally, the actual lattices that have been developed are constrained by the accelerator structure length and modularity and thus the lattice tends to change in steps. Regardless, this simple parameterization can model most lattices quite well. As an example, the horizontal $\beta$-function for the NLC linac is plotted in Fig. 1 . The NLC linac is designed to accelerate the beams from $8 \mathrm{GeV}$ to $500 \mathrm{GeV}$. The lattice consists of roughly 350 FODO cells with roughly equal horizontal and vertical focusing and is well parameterized with $\alpha=0.33$ and $\overline{\beta_{i}}=8$.

\section{WAKEFIELDS}

Wakefields arise when the electro-magnetic field of a charged particle beam interacts with the surrounding vacuum chamber and then act back on the beam itself. Assuming an ultra-relativistic beam, the fields generated by a particle 
can only affect the trailing particles. It is important to note that the 'wakefield' is not the instantaneous force seen by a beam but is an integrated quantity describing the net effect of the induced fields assuming that the beam trajectory is unperturbed. In a periodic structure, the integration length is that of the structure periodicity while, in an aperiodic structure, the integration length extends to infinity.

This causes a significant difference in the high frequency behavior of the wakefields due to a single cavity and that from an infinitely periodic structure. This difference can be important for bunches short compared to the cavity iris radius. In particular, for short bunches, the longitudinal and transverse wakefield of a periodic structure are constant and linear in distance $z$, while those for a single cell scale as $1 / \sqrt{z}$ and $\sqrt{z}$, respectively.

The transition from the single cavity regime to the periodic structure occurs after $a^{3} \sqrt{\pi} / \sigma_{z}^{3 / 2} L^{3 / 2}$ cells, where $a$ is the iris radius and $L$ is the length of a cell $[18,19]$. In most cases of interest, the bunch is sufficiently long that wakefield more closely resembles that of an infinitely period structure than that due to a single cavity; this is even true in the TESLA FEL linac where the bunch is very short compared to the cavity radius [20].

Thus, to estimate the wakefields, we will use a parameterization for a periodic structure, similar to that described in Ref. [21], which is based on the theoretical short-range dependence plus an empirical term to describe the behavior at longer distances:

$$
W_{\|}(z) \approx \frac{Z_{0} c}{\pi a \sqrt{a^{2}+8 z \lambda}}(V / C / m)
$$

where $Z_{0}$ and $c$ are the impedance of free space and speed of light and $a$ and $\lambda$ are the iris radius of the structure and the rf wavelength. Similarly, the short-range transverse wakefield can be written

$$
W_{\perp}(z) \approx \frac{2 Z_{0} c z}{\pi a^{3} \sqrt{a^{2}+5 z \lambda}} \cdot\left(V / C / m^{2}\right)
$$

It should be noted that with short bunches the transverse kick-factor for a periodic structure scale as $\sigma_{z}$-this is reduced for longer bunches because of the curvature of the wakefield and is sometimes approximated as $\sqrt{\sigma_{z}}$.

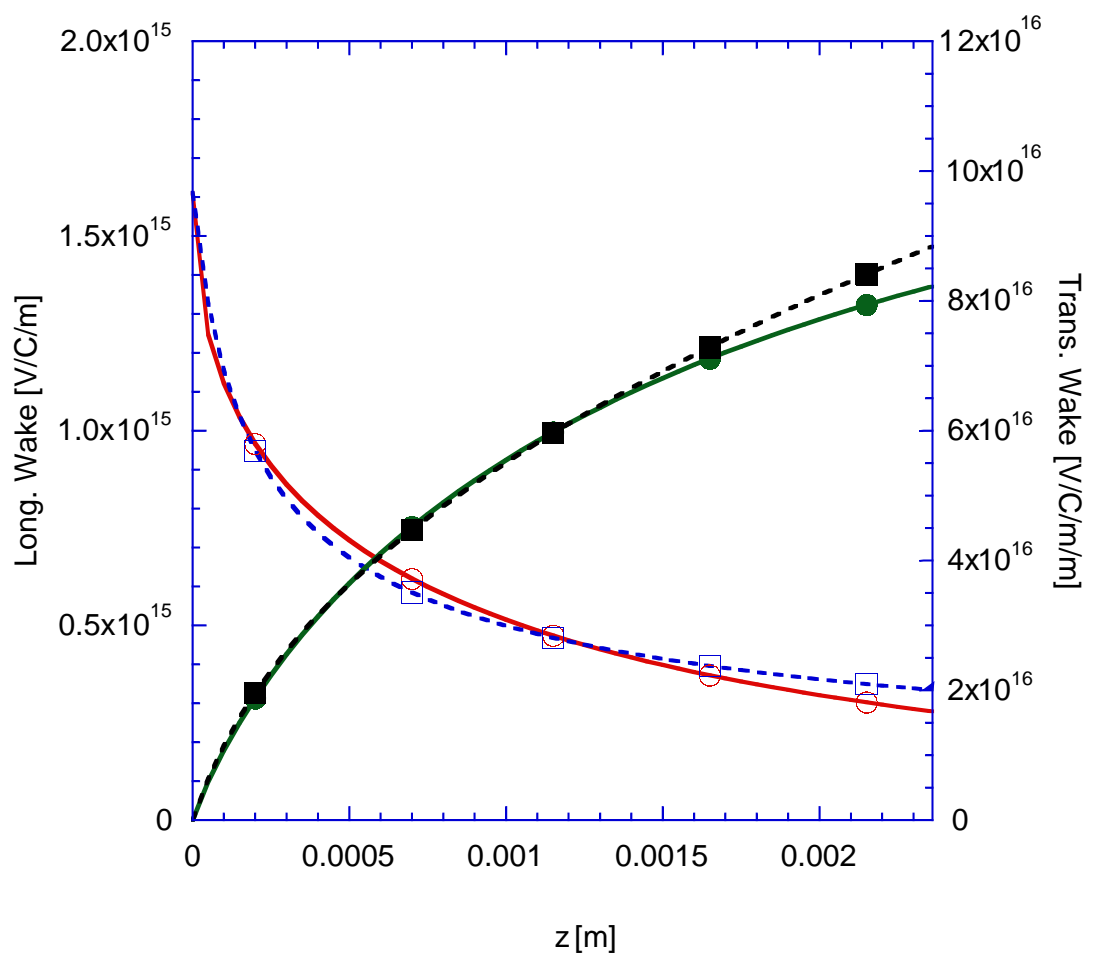

FIG. 2. Short range longitudinal (open circles) and transverse wakefields (solid circles) in the NLC X-band accelerator structures along with the approximations of Eqs. (22) (open squares) and (23) (solid squares).

There are a number of more complex parameterizations [22-24] but these models have been verified to yield good results over the range $s \lesssim a / 4$ and $0.1 \lesssim a / \lambda \lesssim 0.2$. As an example, the short range longitudinal and transverse 
wakefields in the NLC linac are plotted in Fig. 2 along with the approximation of Eqs. (22) and (23); the NLC accelerator structures operate at $11.424 \mathrm{GHz}$ where $a / \lambda=0.18$ and $\lambda=2.625 \mathrm{~cm}$.

The long-range longitudinal and transverse wakefields are dominated by the fundamental monopole and dipole modes. The amplitude of the wakefields scale as:

$$
W_{\|} \sim \frac{1}{a} \quad W_{\perp} \sim \frac{1}{a^{3}}
$$

however both have complicated dependences on other details of the cavity design. A full parameterization of the long-range wakefields is beyond the scope of this paper. However, we note that, when considering a multibunch beam, compensation of the long-range longitudinal wakefield, referred to as beam loading, is an essential part of the accelerator design and thus, in the remainder of this paper, we will assume that the loading is compensated with a residual rms energy error of $\sigma_{\delta L R}$. Similarly, reduction of the long-range transverse wakefield is usually essential to prevent the multi-bunch beam breakup (BBU) instability and, in the remainder of this paper, we will assume that multi-bunch BBU is not a limitation. Given these assumptions, we will describe the effects of the long-range wakefields on the projected beam emittance in Section IX.

\section{LONGITUDINAL PHASE SPACE}

The single bunch longitudinal phase space is determined by the rf acceleration and the short-range longitudinal wakefields. If the longitudinal wakefields are significant, typically the rf phase is chosen to partially cancel the correlated energy spread that is introduced. Control of this correlated energy spread is usually needed for BNS damping - discussed in the next section - and to provide a beam that is within the acceptance of the downstream application.

Because the short-range longitudinal wakefield decreases with distance, the energy spread introduced by the longitudinal wakefields tends to be larger for shorter bunches. In contrast, the compensating energy spread that can be generated by running off-crest of the accelerating if is roughly proportional to the bunch length. This sets a limit on the minimum bunch length for a given maximum rf phase offset and maximum final energy spread. Using a simple uniform beam model with a length of $2.35 \sigma_{z}$, we can estimate the residual rms energy spread after compensation. For a Gaussian beam, we find:

$$
\sigma_{\delta} \approx\left|\frac{(1+0.25 i) 4 \pi \epsilon_{0} N r_{e} \overline{W_{\|}}-2.35 \sigma_{z} \kappa_{r f} G \sin \phi_{r f}}{2.35 G \cos \phi_{r f}}\right| .
$$

In Eq. (25), the first term is the energy spread induced by the wakefield while the second is the effect of the rf acceleration. Here, the length $2.35 \sigma_{z}$ is chosen to fit the limiting behavior at large rf phase angles and $\overline{W_{\|}}$is the weighted average of the longitudinal wakefield over the $\pm 1.17 \sigma_{z}$ and can be well approximated as:

$$
\overline{W_{\|}} \approx 0.76 W_{\|}\left(1.3 \sigma_{z}\right)
$$

Finally, the imaginary coefficient $(1+0.25 i)$ is fit to the results of simulations and approximates the minimum rms energy spread attainable with a Gaussian bunch. 


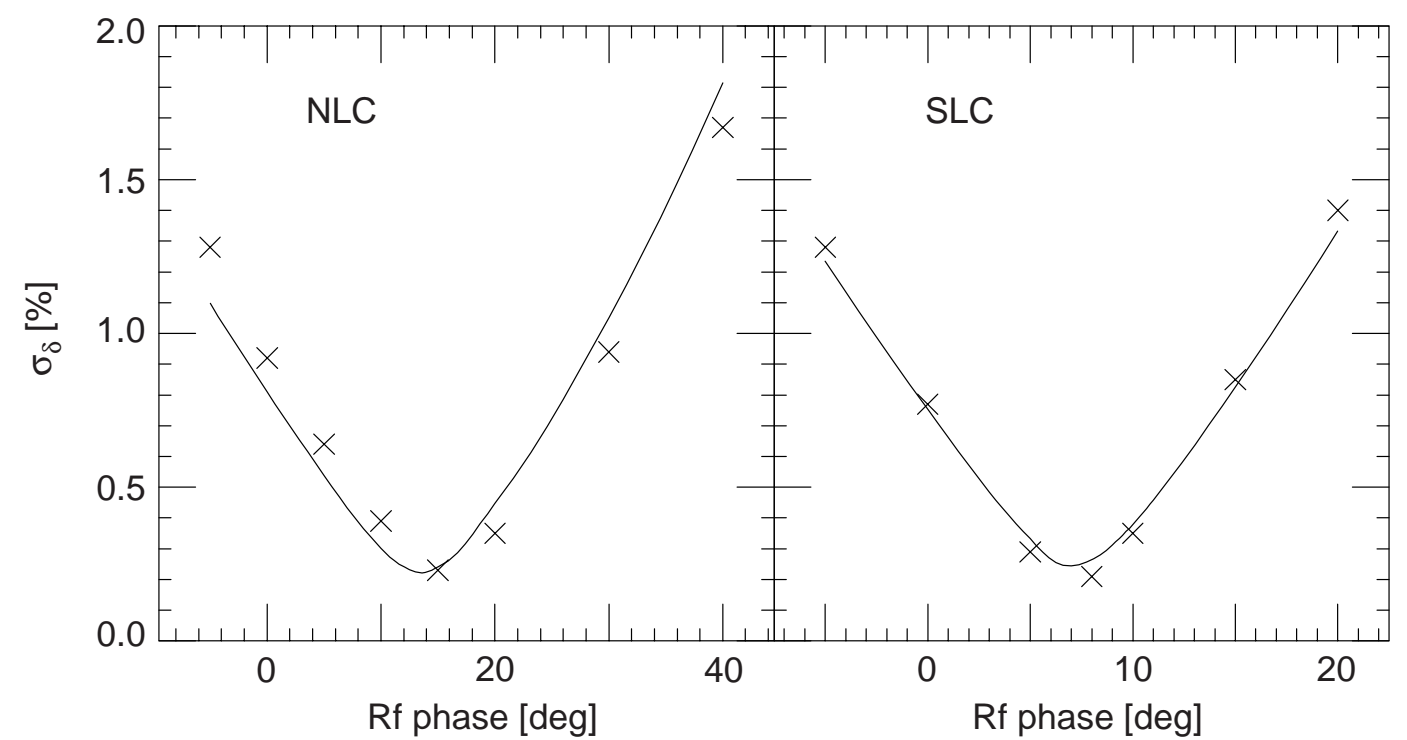

FIG. 3. Rms energy spread versus average rf phase in the NLC and SLC linacs (solid) compared with Eq. (25) (crosses); the NLC linac has $N=1 \times 10^{10}, \sigma_{z}=140 \mu \mathrm{m}, G=57 \mathrm{MV} / \mathrm{m}$, and $11.424 \mathrm{GHz}$ rf structures having an $a / \lambda=0.18$ while the SLC linac has $N=3 \times 10^{10}, \sigma_{z}=1 \mathrm{~mm}, G=17 \mathrm{MV} / \mathrm{m}$, and $2.856 \mathrm{GHz} \mathrm{rf}$ structures having an $a / \lambda=0.12$.

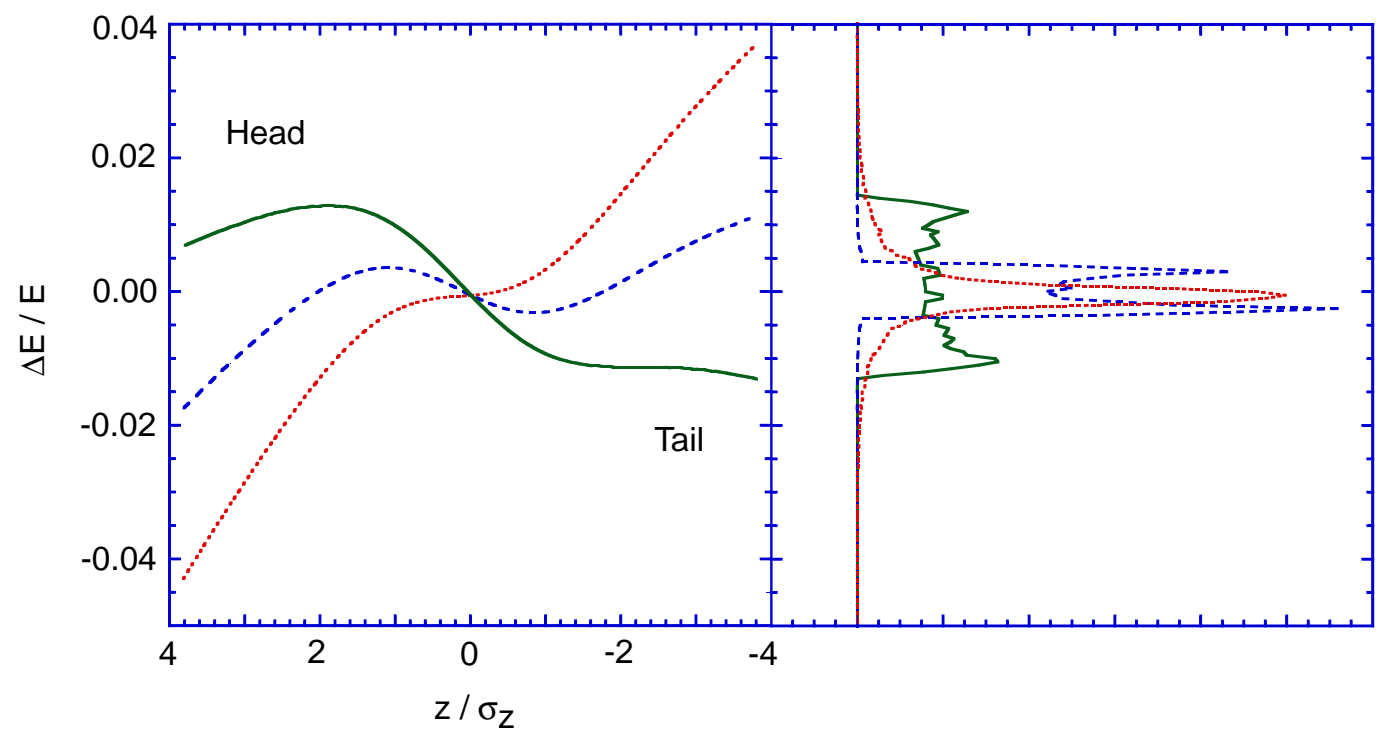

FIG. 4. Longitudinal phase space at the end of the NLC X-band linac when operating with an $\mathrm{rf}$ phase of $0^{\circ}$ (solid), $10^{\circ}$ (dashes), and $20^{\circ}$ (dots) to partially compensate the longitudinal wakefield with the projections of the distributions on the energy axis to the right.

In Fig. 3, we compare the rms energy spread calculated from simulation in the NLC and SLC linacs with the results of Eq. (25). In the NLC linac, the bunch consists of $N=1 \times 10^{10}$ particles and the bunch length is $\sigma_{z}=140 \mu \mathrm{m}$ while, in the SLC linac, bunch consists of $N=3 \times 10^{10}$ particles and the bunch length is $\sigma_{z}=1 \mathrm{~mm}$. In both cases, Eq. (25) agrees well with the results of the simulations and accurately predicts the location of the minimum energy spread at the level of a few degrees.

In Fig. 4, the longitudinal phase space at the end of the NLC linac is plotted assuming that the rf is phased for on-crest operation and $10^{\circ}$ and $20^{\circ}$ off-crest to partially compensate the short-range longitudinal wakefield. Note that at an rf phase of $20^{\circ}$, the core of the beam has a narrow energy spread but the beam also has significant off-energy tails. In contrast, the total energy spread and, as can be seen in Fig. 3, the rms energy spread are smaller at an rf phase of $10^{\circ}$. Since the increased rf phase angle also decreases the acceleration, the optimal phase angle is usually chosen to minimize the full width of the distribution and is accurately estimated by setting the real part of Eq. (25) 
to zero.

Finally, we should note that there are other techniques of reducing the energy spread introduced by the longitudinal wakefield instead of simply adjusting the average rf phase. One example is to shape the longitudinal beam distribution [25]; this technique has been used in the SLC linac to reduce the energy spread by a factor of $2 \sim 3$ [26]. Another approach is to rotate the bunch in longitudinal space by more than 90 degrees part way through linac. This flips the sign of the energy correlation and then the longitudinal wakefield can remove the energy spread that it had previously introduced [27]; this technique allows control of the energy spread of very short bunches where the rf phase has little effect.

\section{BNS DAMPING AND AUTOPHASING}

If the beam performs a coherent betatron oscillation without compensation of the short-range dipole wakefield, the wakefields induced by the head of the bunch will resonantly drive the tail of the bunch. This leads to emittance dilution and amplification of the incoming trajectory jitter known as single-bunch beam break-up (bbu) [28].

BNS damping [29] is a technique of controlling the single-bunch beam break-up due to the transverse wakefields by generating a focusing variation along the bunch which can be implemented using RF quadrupoles [30] or by adding a correlated energy deviation along the bunch as was originally proposed. The BNS technique has been studied by many authors using both analytic techniques [31-33] as well as computer simulation.

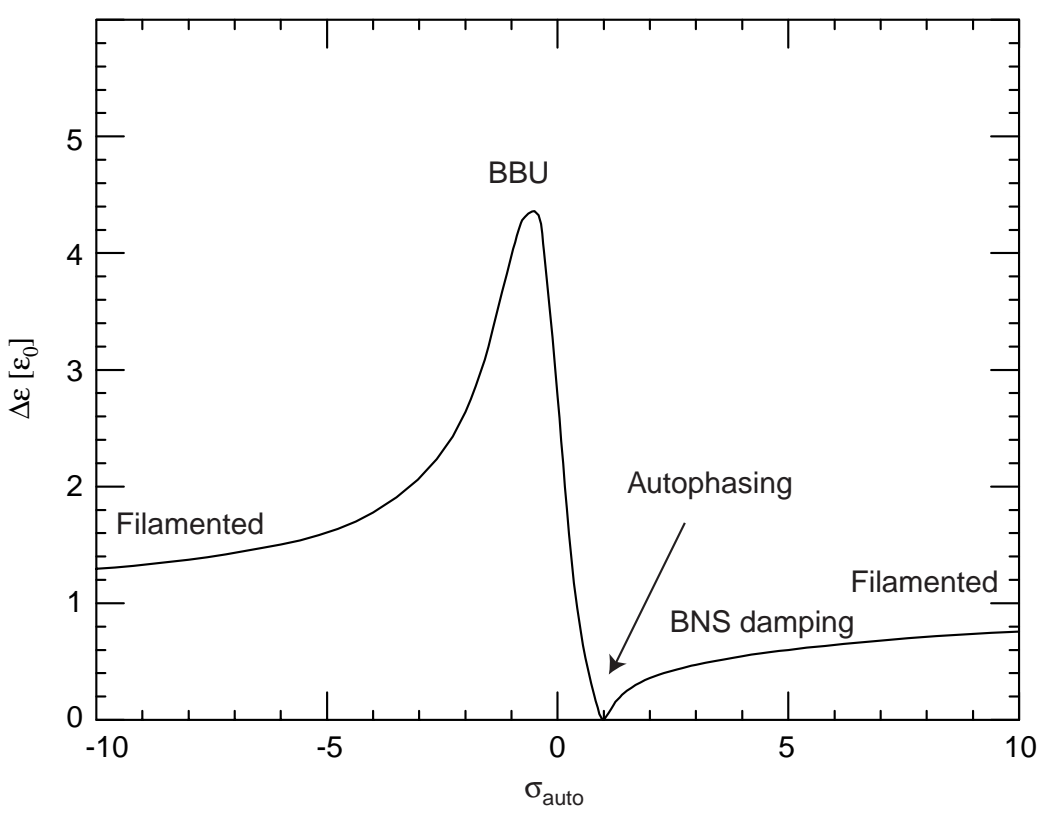

FIG. 5. Emittance growth for a $\sqrt{2 / \beta} \sigma$ initial transverse offset versus correlated energy spread in units of the optimal energy spread for 'autophasing' after the first $10 \%$ of the NLC linac; note the different regimes with filamentation at $\sigma_{\delta} \gg \sigma_{a u t o}$ or $\ll \sigma_{\text {auto }}$, beam breakup when $\sigma_{\delta} \sim 0$, BNS damping when $\sigma_{\delta}>\sigma_{\text {auto }}$, and autophasing when $\sigma_{\delta}=\sigma_{\text {auto }}$.

Empirically, BNS damping can be separated into four regions: filamentation where the correlated energy spread is so large that the coherent oscillation rapidly decoheres with a corresponding increase in the beam emittance; the break-up region where the transverse wakefields dominate; 'autophasing' [34] where the wakefield is exactly compensated by the correlated energy spread and the coherent oscillation propagates without emittance dilution; and, the damping region where the wakefield acts to damp the oscillation without a corresponding increase in emittance. This is illustrated in Fig. 5 where the rms emittance growth is plotted versus the correlated rms energy spread in units of that needed for perfect autophasing. The emittance dilution is calculated for a bunch having an initial offset equal to $\sqrt{2 / \beta} \sigma$ after the first $10 \%$ of the NLC linac, a distance of roughly $1 \mathrm{~km}$. Note that when the energy spread is equal to the autophasing energy spread, the emittance growth goes to zero, but when the energy spread is large, the initial oscillation filaments (phase mixes) leading to a unit increase in the beam emittance-filamentation is discussed in Section VIII A.

The autophasing condition for a rigid offset of a bunch can be immediately derived from Eq. (4). The goal is to choose $\delta(z)$ so that the chromatic behavior of the quadrupoles compensates the effect of the wakefield. In the smooth 
approximation, where both $K$ and $W_{\perp}$ are constant or slowly varying, exact cancellation is possible:

$$
\delta_{\text {auto }}(z)=\frac{4 \pi \epsilon_{0} N r_{e}}{K \gamma_{0}} \int_{z}^{\infty} W_{\perp}\left(z^{\prime}-z\right) \rho\left(z^{\prime}\right) d z^{\prime}
$$

and the ideal autophasing energy spread for a gaussian bunch in the NLC linac is shown in Fig. 6.

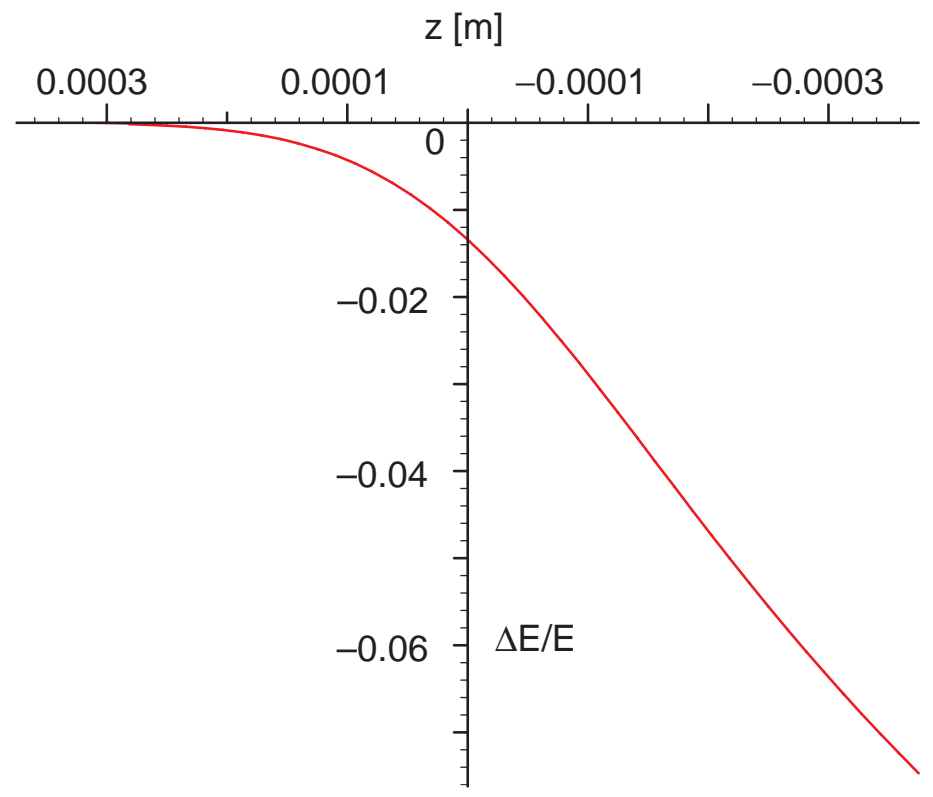

FIG. 6. Ideal autophasing energy spread for a gaussian bunch in the beginning of the NLC linac with a bunch length of $\sigma_{z}=125 \mu \mathrm{m}$ and $N=1 \times 10^{10}$; the head of the bunch is to the left-note the nonlinear correlation which is difficult to generate.

Unfortunately, the nonlinear variation in the desired autophasing energy spread is difficult to generate with the longitudinal wakefields and the rf phase; for an example of the typical longitudinal phase space, see Fig. 4 . Furthermore, in the more realistic case of a strong focusing lattice, the autophasing condition does not provide local compensation of the wakefield although it can still cancel the effect of the transverse wakefield when averaged over a coherent betatron oscillation. In this case, an approximate autophasing condition, which describes the variation of the correlated energy deviation per $\sigma_{z}$ about the bunch center, can be estimated:

$$
\sigma_{\text {auto }} \approx\left(\pi \epsilon_{0} N r_{e} W_{\perp}\left(2 \sigma_{z}\right)\right) \frac{\bar{\beta} L_{\text {cell }}}{4 \gamma \tan \left(\psi_{c} / 2\right)}
$$

where $\bar{\beta}$ is defined in Eq. (18). Note that $\sigma_{\text {auto }}$ is a monotonically decreasing function of the phase advance per cell (between $0^{\circ}$ and $180^{\circ}$ ) which is one reason to choose a relatively large phase advance per cell. In addition, assuming the linac scaling described earlier, the energy spread required for autophasing scales as $\gamma^{2 \alpha-1}$ and is constant along the linac for $\alpha=0.5$.

This autophasing condition provides a useful measure of the importance of the wakefields on the beam dynamics in the linac. In particular, the wakefield strength parameter $\eta$ defined in Ref. [28], is proportional to $\sigma_{\text {auto }}$ times the chromaticity of the linac and, as will be seen in Sec. VIIIB, $\sigma_{\text {auto }}$ can be used to parameterize the emittance dilution. In the NLC linac, Eq. (28) specifies and energy variation of $1.9 \%$ per $\sigma_{z}$ at the beginning of the linac-this is roughly twice the variation seen in Fig. 4 at an $\mathrm{rf}$ phase of $0^{\circ}$. In contrast, the autophasing energy spread for the SLC linac was roughly $4.5 \%$ per $\sigma_{z}$ despite the much weaker wakefields; the difference arises because of the smaller bunch charge and shorter bunches as well as the stronger focusing in the NLC linac.

Finally, we should note that this autophasing condition was derived for a rigid offset of the bunch. A similar condition can be found for a bunch offset generated by a misaligned accelerator section. Here, the offset of the bunch is a function of $z$, the longitudinal position within the bunch. In this case, the 'autophasing' condition, i.e., the condition to preserve this $x-z$ correlation as the bunch propagates down the linac, is roughly $\frac{1}{6}$ the magnitude of that for a rigid offset [35]. 


\section{SINGLE-BUNCH EMITTANCE DILUTIONS}

At this point, we can discuss the primary sources of emittance dilution. In doing so, we will assume that the beam is close to the autophasing condition and thus the propagation of a betatron oscillation can be described with the design optical functions which is not case when far from the autophasing condition [36].

The primary sources of dilution are transverse wakefields, dispersive errors, and betatron coupling; we will also present estimates for the effect of RF deflections and beam-gas scattering. With the exception of the beam-gas scattering and, at very high energy, incoherent synchrotron radiation, all of the dilutions encountered in a linac are conservative dilutions. A conservative emittance dilution arises when the transverse or longitudinal degrees of freedom become coupled. In this case, the six-dimensional emittance is conserved but the projected emittances, which are frequently the relevant values, are increased. Specifically, the principal sources of dilution and the couplings are [37]:

- Transverse wakefields $-z \rightarrow\left(y, y^{\prime}\right)$

- Dispersive errors $-\delta \rightarrow\left(y, y^{\prime}\right)$

- Betatron coupling - $\left(x, x^{\prime}\right) \rightarrow\left(y, y^{\prime}\right)$

- $\mathrm{RF}$ deflections $-z \rightarrow\left(y, y^{\prime}\right)$

It can easily be shown that coupling of two planes always increases the smaller of the two projected emittances from the uncoupled value. However, because the emittance dilutions are conservative, they can be corrected provided the dilution has not filamented (phase mixed). For example, emittance correction techniques were used in the SLC to reduce the emittance dilution from $1000 \%$ to about $100 \%$ [38].

The effectiveness of the emittance correction techniques will be discussed in Section XII when we discuss the effect of more complex correction techniques. However, in this section, we will calculate the dilutions assuming 1-to-1 trajectory correction where the beam trajectory is corrected to minimize the readings of Beam Position Monitors (BPMs) located either all the quadrupoles or just at the focusing quadrupoles. These dilutions are sometimes referred to as 'bare machine dilutions' since minimal correction has been applied.

\section{A. Injection Errors and Filamentation}

The first dilutions to consider are those due to injection errors. If the beam is injected with a trajectory error, it will perform a betatron oscillation along the linac. Provided the beam is close to the autophasing condition, the effect of the transverse wakefields will be minimal. However, if the injected beam has an uncorrelated energy spread, it will start to 'filament' because of the chromatic dependence of the phase advance-the degree of filamentation depends upon the magnitude of the energy spread and the chromaticity of the lattice which is given in Eq. (19). Alternately, if the magnitude of the correlated energy spread is much greater than the autophasing energy spread, the beam will also filament as described in Section VII. In either case, the betatron oscillation will decohere and the projected emittance of the beam will increase.

The emittance dilution due to the filamented injection trajectory error can be expressed as [39]:

$$
\Delta \gamma \epsilon \approx \frac{\gamma}{2}\left(\frac{\left(1+\alpha_{y}^{2}\right)}{\beta_{y}} y_{0}^{2}+2 \alpha_{y} y_{0} y_{0}^{\prime}+\beta_{y} y_{0}^{\prime 2}\right)
$$

where $y_{0}$ and $y_{0}^{\prime}$ are the injection trajectory errors and $\beta_{y}$ and $\alpha_{y}$ are the lattice functions at the injection point.

A similar effect will arise if the beam is injected with a dispersive mismatch except that beam performs a quadrupole oscillation rather than a dipole oscillation. The emittance dilution is again given by Eq. (29) with the substitution $y_{0} \rightarrow \sigma_{\delta} \eta_{0}$ and $y_{0}^{\prime} \rightarrow \sigma_{\delta} \eta_{0}^{\prime}[16]$ where $\eta_{0}$ and $\eta_{0}^{\prime}$ are the injected dispersive errors and $\sigma_{\delta}$ is the energy spread in the beam.

Finally, another similar effect will arise if the beam is injected with a betatron mismatch - it should be noted that a long linac has well defined quasi-periodic 'natural' lattice functions, similar to a storage ring, where the optical variations $d \beta / d \delta$ and $d \alpha / d \delta$ are minimum [12]. In this case, the emittance dilution can be parameterized in terms of $B_{\text {mag }}$ [40]

$$
\Delta \gamma \epsilon \approx \gamma \epsilon\left(B_{\text {mag }}-1\right)
$$

and 


$$
B_{\text {mag }} \equiv \frac{1}{2}\left[\left(\frac{\beta}{\beta_{\star}}+\frac{\beta_{\star}}{\beta}\right)+\left(\alpha \star \sqrt{\frac{\beta}{\beta_{\star}}}-\alpha \sqrt{\frac{\beta_{\star}}{\beta}}\right)^{2}\right] .
$$

Here, the subscript $\star$ is used to denote the parameters describing the beam, $\beta_{\star}=\sigma^{2} / \epsilon$ and $\alpha_{\star}=-\left\langle y y^{\prime}\right\rangle / \epsilon$, while the lattice parameters without subscript denote the natural lattice functions of the linac.

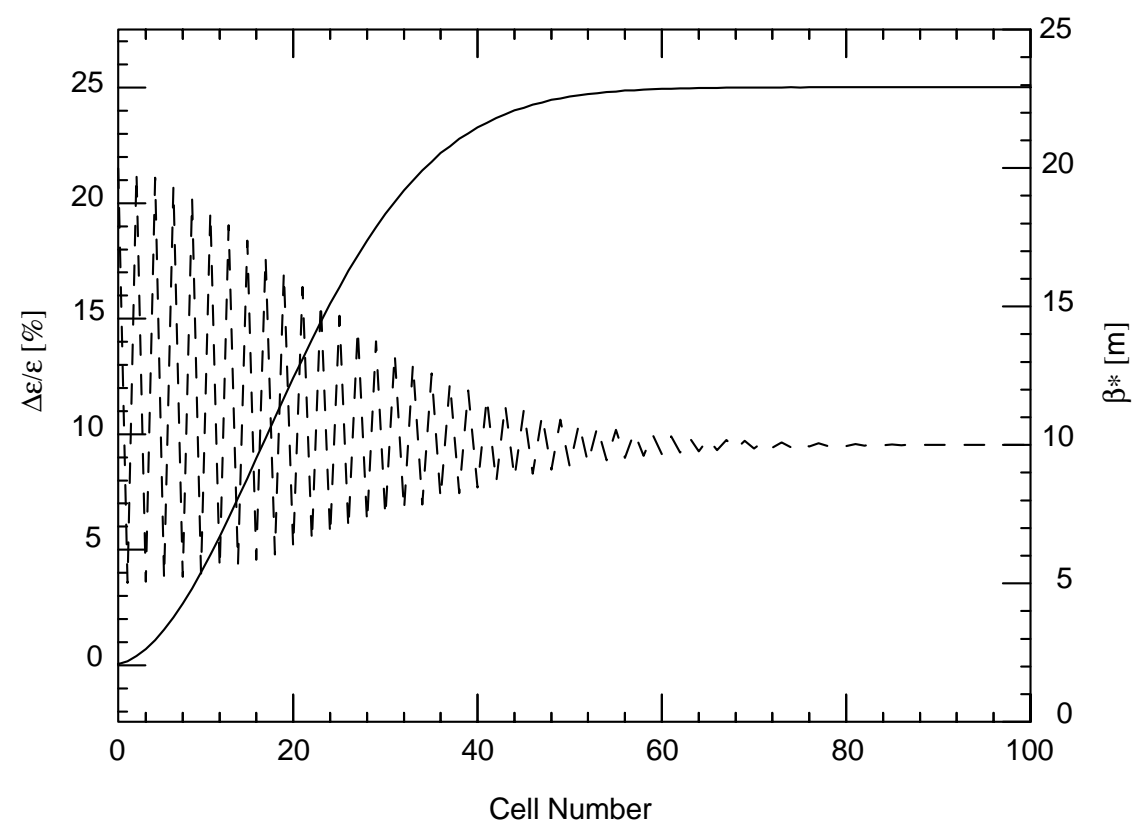

FIG. 7. Filamentation of a mismatched beam with a $1 \%$ energy spread in $10090^{\circ}$ FODO cells and an initial $\beta_{\star}=2 \beta$; the emittance dilution (solid) increases as the beam filaments while the beam beta-function $\beta_{\star}$ (dashes) approaches the periodic lattice beta-function.

This is illustrated in Fig. 7 where a beam with a betatron mismatch of $\beta_{\star}=2 \beta$ and a $1 \%$ energy spread is injected into a 100 cell FODO lattice, similar to the lattice of the NLC linac. The beam rapidly filaments leading to emittance dilution (solid) while the parameters describing the beam ellipse $\beta_{\star}$ and $\alpha_{\star}$ approach the lattice parameters. The $\beta_{\star}$ (dashes) is plotted at the focusing quadrupoles where $\beta=10 \mathrm{~m}$.

Note that although Eqs. (29) and (30) describe the evolution of the beam rms emittance, the beam distribution will also evolve during the filamentation process. For example, if a gaussian beam is injected with a trajectory error which subsequently filaments, the resulting distribution could be symmetric but hollow, i.e. having a minimum on axis [39]. Alternately, if a betatron mismatch filaments, the resulting distribution becomes much more sharply peaked [41,42].

\section{B. Trajectory Misalignments}

Next, we will consider the dilutions due to a corrected trajectory. The trajectory errors will have two effects: the offsets in the quadrupoles will generate anomalous dispersion and the offsets in the accelerator structures will cause wakefield deflections. These dilutions will depend on the rms misalignment of the elements about a 'smoothed' alignment path. This arises because only errors with wavelengths comparable to the betatron wavelength have a significant effect on the beam emittance; this is illustrated in Fig. 8 where we have plotted the dispersion arising after steering the beam to BPMs which have a periodic misalignment. At wavelengths much longer than the betatron wavelength, the dispersion decreases inversely with the square of the wavelength; the results are analogous for dilutions due to wakefields. 


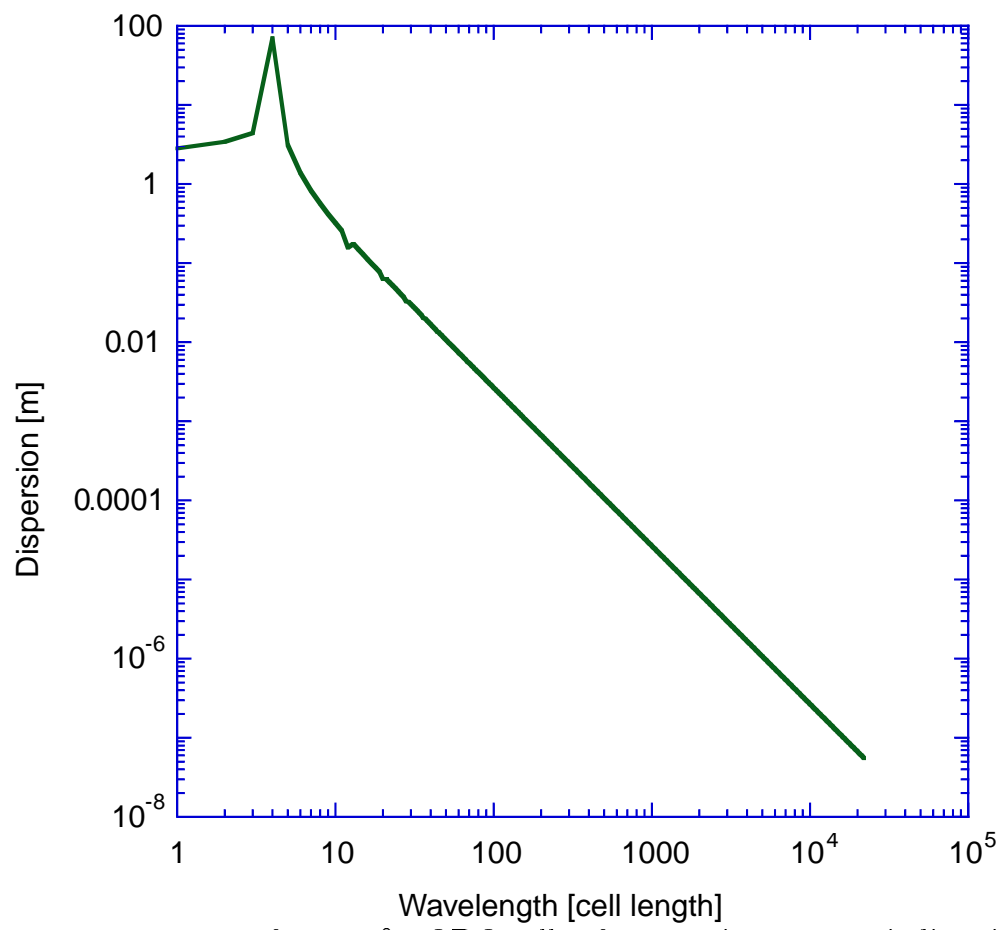

FIG. 8. Dispersion arising at the end of $10090^{\circ}$ FODO cells after steering to a periodic misalignment with a wavelength in units of the cell length; note that the betatron wavelength in a $90^{\circ}$ cell is equal to the length of four cells which is where the dispersion peaks.

In this section, we will assume that the accelerator structures are aligned properly and only the BPMs and quadrupoles are misaligned relative to the smooth fiducial. Thus, the trajectory is deflected with either quadrupole movers or dipole correctors to follow the BPM misalignments and the trajectory will be offset in the accelerator structures and quadrupoles. We will further assume that the beam is close to the autophasing condition. In this case, the emittance dilution will be due to three different effects:

1. dispersive errors due to a constant energy spread which might arise from improper beam loading compensation or deviations from the ideal autophasing energy spread,

2. dispersive errors due to an injected energy spread which adiabatically damps as the beam is accelerated,

3. dispersive errors due to the autophasing energy spread and wakefields due to the offsets in the accelerator structures, the two of which partially cancel each other.

We will consider two different correction schemes: '1-to-1' trajectory correction where the trajectory is corrected to minimize the readings of Beam Position Monitors (BPMs) located at all of the quadrupoles and '1-to-1' correction where the trajectory is corrected to minimize the BPM reading only at the focusing quadrupoles. More complicated trajectory correction algorithms are described in Section XII.

First, consider the case where the trajectory is corrected to minimize the BPM readings at every quadrupole. Assuming that the beam is close to the autophasing condition, the emittance dilution due to dispersion from a constant energy spread $\delta_{c}$ is

$$
\Delta \gamma \epsilon \approx 4\left\langle y_{B P M}^{2}\right\rangle \delta_{c}^{2} \frac{\tan \frac{\psi_{c}}{2}}{L_{\text {cell } i}^{2}} \frac{\gamma_{i}^{2}}{(1-\alpha) G}\left[\left(\frac{\gamma_{f}}{\gamma_{i}}\right)^{2-2 \alpha}-1\right]
$$

where the subscripts $i$ and $f$ are used to denote initial and final, and the value at $\alpha=1$ is given by the limit:

$$
\lim _{\alpha=1} \frac{\left(x^{\alpha}-1\right)}{(1-\alpha)}=\ln (x) \quad .
$$

If instead, the trajectory is only corrected to minimize the BPM readings at the focusing quadrupoles and the defocusing quadrupoles are well aligned, the dilution is 


$$
\Delta \gamma \epsilon \approx 8\left\langle y_{B P M}^{2}\right\rangle \delta_{c}^{2} \frac{\tan ^{3} \frac{\psi_{c}}{2}\left(1-\sin \frac{\psi_{c}}{2}\right)}{L_{\text {cell } i}^{2}} \frac{\gamma_{i}^{2}}{(1-\alpha) G}\left[\left(\frac{\gamma_{f}}{\gamma_{i}}\right)^{2-2 \alpha}-1\right] .
$$

Note that although the dependence on the phase advance differs, the dilution expected when only correcting at the focusing quadrupoles can be significantly less than when correcting at all the quadrupoles. The difference is given by the factor:

$$
2 \tan ^{2}\left(\psi_{c} / 2\right)\left(1-\sin \left(\psi_{c} / 2\right)\right)
$$

which ranges from 0.2 at $45^{\circ}$ per cell to 1 at $180^{\circ}$ per cell. This arises because the trajectory offset is demagnified in the defocusing quadrupoles, leading to smaller offsets on average. Finally, the effect of misalignments of the defocusing quadrupoles can be included by multiplying Eq. (34) by:

$$
1+\left(\frac{y_{Q D}}{y_{B P M}}\right)^{2}\left(1-\sin \frac{\psi_{c}}{2}\right)^{2}
$$

where $y_{Q D}$ is the misalignment of the defocusing quadrupoles.

Next, we can consider the effect of an initial uncorrelated energy spread which will adiabatically damp as $1 / \gamma$ while beam is accelerated. In this case, assuming that the trajectory is corrected to zero BPMs at the focusing quadrupoles, the emittance dilution due to dispersion will be:

$$
\Delta \gamma \epsilon \approx 8\left\langle y_{B P M}^{2}\right\rangle \delta_{0}^{2} \frac{\tan ^{3} \frac{\psi_{c}}{2}\left(1-\sin \frac{\psi_{c}}{2}\right)}{L_{\text {cell } i}^{2}} \frac{\gamma_{i}^{2}}{\alpha G}\left[1-\left(\frac{\gamma_{i}}{\gamma_{f}}\right)^{2 \alpha}\right]
$$

where $\delta_{0}$ is the injected energy spread. One can find the expression for correcting at all of the quadrupoles by dividing by the factor in Eq. (35) and the effect of misalignments of the defocusing quadrupoles is given by Eq. (36).

Finally, we can consider the effect of the autophasing energy spread and the wakefields. The autophasing energy spread is chosen to eliminate the emittance dilution due to a coherent betatron oscillation however the cancellation between the dispersive effects and the wakefields will not be perfect for a corrected trajectory where the beam is steered to follow the BPM misalignments. As discussed in Section VII, the ideal autophasing energy spread scales as $\gamma^{2 \alpha-1}$ when the beam is accelerated. Assuming that this condition is maintained and assuming that the trajectory is corrected to zero BPMs at all the quadrupoles, the expected emittance dilution will be:

$$
\Delta \gamma \epsilon \approx\left\langle y_{B P M}^{2}\right\rangle\left(\pi \epsilon_{0} N r_{e} W_{\perp}\left(2 \sigma_{z}\right)\right)^{2} \frac{L_{\text {cell } i}^{2}}{32 \alpha G} f(\psi)\left[\left(\frac{\gamma_{f}}{\gamma_{i}}\right)^{2 \alpha}-1\right]
$$

where $f$ is a factor that depends on the phase advance:

$$
f(\psi)=\frac{1}{18}\left[\frac{1+46 \cos ^{2} \frac{\psi_{c}}{2}-11 \cos ^{4} \frac{\psi_{c}}{2}}{\sin ^{3} \frac{\psi_{c}}{2} \cos \frac{\psi_{c}}{2}}\right] .
$$

Note that when $\alpha=\frac{1}{2}$ and the autophasing energy spread is constant along the linac, Eq. (38) has a form similar to Eq. (32) but tends to be smaller due to the cancellation of the dispersive errors by the wakefield kicks.

A similar expression can be found when only correcting to zero the BPM's at the focusing quadrupoles, except, in this case, the phase advance factor $f$ is:

$$
f_{Q F}(\psi)=2 \tan ^{2} \frac{\psi_{c}}{2}\left(1-\sin \frac{\psi_{c}}{2}\right)\left[\frac{25+24 \sin \frac{\psi_{c}}{2}+12 \sin \frac{\psi_{c}}{2} \cos ^{2} \frac{\psi_{c}}{2}+7 \cos ^{2} \frac{\psi_{c}}{2}+4 \cos ^{4} \frac{\psi_{c}}{2}}{288 \sin ^{3} \frac{\psi_{c}}{2} \cos \frac{\psi_{c}}{2}}\right]
$$

where the first factor is the same as that found before, Eq. (35), and the term within the square brackets has a behavior similar to Eq. (39) but is smaller at low phase advance and larger at high phase advance.

In both Eqs. (39) and (40), the complicated dependence on the phase advance arises from the different sensitivities of the dispersive and the wakefield terms. In both cases, the phase advance factor $f$ becomes large when $p s i=0^{\circ}$ or $180^{\circ}$ where the wakefield or dispersive terms dominate. When correcting at all the quadrupoles, the factor $f$ is a minimum at roughly $160^{\circ}$ per cell while, when correcting at just the focusing magnets, the factor $f$ is a minimum at roughly $100^{\circ}$ per cell. 


\section{Accelerator Structure Misalignments}

The next dilution to consider is that due to accelerator structure misalignments. Like the BPM errors just discussed, we assume that the errors are random about a smoothed trajectory. In this case, the emittance dilution can be estimated as:

$$
\Delta \gamma \epsilon \approx\left\langle y_{a}^{2}\right\rangle\left(\pi \epsilon_{0} N r_{e} W_{\perp}\left(2 \sigma_{z}\right)\right)^{2} \frac{L_{\mathrm{acc}} \bar{\beta}_{i}}{2 \alpha G}\left[\left(\frac{\gamma_{f}}{\gamma_{i}}\right)^{\alpha}-1\right]
$$

where $L_{\text {acc }}$ is the length of the accelerator structures and is assumed to be constant along the length of the linac and all other quantities have been defined.

Equation (41) assumes that the alignment of the individual structures is purely random. A related dilution will occur if all the structures between a pair of quadrupoles have a systematic misalignment; such an error would likely occur when mechanically aligning the structures since the quadrupoles frequently provide the reference for the alignment. In this case, the dilution can be estimated as:

$$
\Delta \gamma \epsilon \approx\left\langle y_{a}^{2}\right\rangle\left(\pi \epsilon_{0} N r_{e} W_{\perp}\left(2 \sigma_{z}\right)\right)^{2} \frac{L_{\mathrm{cell} i} \bar{\beta}_{i}}{4 \alpha G}\left[\left(\frac{\gamma_{f}}{\gamma_{i}}\right)^{2 \alpha}-1\right]
$$

Note that this expression is similar in form to that due to trajectory offsets Eq. (32). In general, this dilution is substantially greater than that due to purely random errors because more structures are contributing.

\section{Betatron Coupling}

In future linear colliders, the vertical emittance is typically much smaller than the horizontal and thus there are tight tolerances on the allowable skew quadrupole fields. Assuming purely random errors, the emittance dilution is $[12]$

$$
\Delta \gamma \epsilon_{y} \approx \gamma \epsilon_{x} 4 N_{c e l l}\left(\frac{\widetilde{K}}{K_{1}}\right)^{2}
$$

where $\tilde{K}$ is the normalized skew quadrupole gradient and $K_{1}$ is the normalized quadrupole gradient. In the case of quadrupole roll errors, the skew field is equal to $\widetilde{K}=2 \Theta_{q} K_{1}$.

Similarly, assuming systematic errors, the dilution can be found to be

$$
\Delta \gamma \epsilon_{y} \approx \gamma \epsilon_{x}\left(\frac{\widetilde{K}_{Q F}+\widetilde{K}_{Q D}}{\left|K_{1}\right|}\right)^{2} \frac{\sin ^{2} N_{c e l l} \frac{\Delta \psi_{c}}{2}}{\sin ^{2} \frac{\Delta \psi_{c}}{2}}
$$

where the subscripts $Q D$ and $Q F$ are used to denote the skew components of the focusing and defocusing quadrupoles and $\Delta \psi_{c}=\psi_{x c}-\psi_{y c}$ is the difference between the horizontal and vertical phase advances per cell. Note that the dilution is proportional to $N_{\text {cell }}^{2}$ when the phase advance difference is small $\Delta \psi_{c} \lesssim 2 / N_{\text {cell }}$ and thus it is standard to split the horizontal and vertical phase advances by a few degrees per cell. Also note that the dilution depends on the sum of the skew component at the focusing and defocusing quadrupoles. Thus, if all elements have the same error, i.e., the same roll or same pole error, the dilution will be small because the errors cancel.

\section{E. RF Deflections}

RF deflections arise if the time varying acceleration field is not oriented in the direction of beam propagation; the misalignment can be due to a misaligned accelerator structure, an angular trajectory through a structure, or asymmetries in a structure such as tilted irises or the couplers. In addition to deflecting the beam, the RF deflections will increase the projected emittance by causing a deflection that is a function of the longitudinal position in the bunch. This emittance dilution is

$$
\Delta \gamma \epsilon \approx\left\langle g^{2}\right\rangle\left(\sigma_{z} \kappa_{r f}\right)^{2} \frac{\bar{\beta}_{i} L_{a c c} G}{\alpha}\left[\left(\frac{\gamma_{f}}{\gamma_{i}}\right)^{\alpha}-1\right]
$$


where $\kappa_{r f}$ is the rf wavenumber and $g$ is the longitudinal-transverse coupling of the deflection which is approximately:

$$
g=\left\{\begin{array}{ll}
\Theta_{a c c} / 2 & \text { structure misalignment } \\
\Theta_{\text {tra }} / 2 & \text { trajectory misalignment } \\
\lesssim \Theta_{\text {iris }} & \text { tilted irises }
\end{array} .\right.
$$

\section{F. Beam-Gas Scattering}

Beam-gas scattering is rarely an issue for the emittance in a linac-instead, the primary effect is to contribute to a halo of large amplitude particles which may cause background or radiation problems. The increase in the rms emittance can be estimated

$$
\Delta \gamma \epsilon \approx n_{\text {gas }} \frac{160 \pi Z(Z+1) r_{e}^{2} \bar{\beta}_{i}}{\alpha G}\left[\left(\frac{\gamma_{f}}{\gamma_{i}}\right)^{\alpha}-1\right]
$$

where $Z$ is the atomic number of the gas and $n_{\text {gas }}$ is the density of the residual gas which, at $20^{\circ} \mathrm{C}$, is about $n_{\text {gas }} \approx 3.22 \times 10^{22} n_{m o l} P\left[m^{-3}\right]$. Here, $n_{m o l}$ is the number of atoms per molecule of gas and $P$ is the vacuum pressure in torr.

As stated, this emittance growth is usually dominated by the large amplitude scattering which creates a halo about the beam. The number of particles scattered out to an amplitude greater than $k$ times the rms beam size can be estimated as

$$
\frac{\Delta N}{N} \approx \frac{n_{\text {gas }}}{k^{2}} \frac{\bar{\beta}_{i}}{\gamma \epsilon} \frac{4 \pi Z^{2} r_{e}^{2}}{\alpha G}\left[\left(\frac{\gamma_{f}}{\gamma_{i}}\right)^{\alpha}-1\right]
$$

\section{MULTI-BUNCH EMITTANCE DILUTIONS}

Like the single-bunch beam break-up, the multi-bunch bbu $[43,44,32]$ leads to an amplification of the incoming trajectory jitter which can make operation of the linac virtually impossible. There are techniques, similar to BNS damping, that can reduce the sensitivity to the multi-bunch bbu $[47,48]$ however, the magnitude of the wakefields are such that, frequently, the only viable solution appears to be direct reduction of the long-range transverse wakefield.

When the wakefield is rapidly decaying, one can obtain an estimate of the multi-bunch bbu by assuming that the wakefield only extends to the immediately following bunch [45]. In this case, the criterion for little or no blowup is:

$$
\left|\frac{2 \pi \epsilon_{0} N r_{e} W_{\perp}\left(\Delta_{b}\right) \bar{\beta}_{i}}{\alpha G}\right|\left(\frac{\gamma_{f}}{\gamma_{i}}\right)^{\alpha}<1,
$$

where $W_{\perp}\left(\Delta_{b}\right)$ is the wakefield at the following bunch.

Most long linacs operate in this regime where the multi-bunch bbu is small just like most linacs operate close to the autophasing condition. In such a situation, one can estimate the increase in projected emittance due to misaligned accelerator structures and a corrected trajectory in a manner very similar to that for the single bunch case. The primary difference is that we have to replace the short-range transverse wakefield with the rms 'sum' wakefield [46]. The 'sum' wakefield is the sum of the wakefields from the preceding bunches at a bunch location assuming all bunches have the same offset:

$$
W_{\text {sum }}(n \Delta s) \equiv \sum_{j=0}^{n} W_{\perp}(j \Delta s) .
$$

and the rms sum wakefield is just the rms of the sum wakefield at the different bunches:

$$
W_{r m s}^{2} \equiv \frac{1}{n_{b}} \sum_{j=0}^{n_{b}-1} W_{\text {sum }}^{2}(j \Delta s)-\left(\frac{1}{n_{b}} \sum_{j=}^{n_{b}-1} W_{\text {sum }}(j \Delta s)\right)^{2} .
$$

Here, $n_{b}$ is the number of bunches and $\Delta s$ is the bunch separation. As stated, one can now use the single bunch emittance dilution formulas presented in Section VIIIC, simply replacing $W_{\perp}\left(2 \sigma_{z}\right)$ with $4 W_{r m s}$.

Similarly, if the beam loading due to the long-range longitudinal wakefield is not perfectly compensated, there will be an energy deviation from bunch-to-bunch. Given such an rms spread in energies, the projected emittance dilution can be estimated from the expressions given in Section VIII B. 


\section{COMPONENT MOTION: JITTER AND STABILITY}

There are three issues that need to be considered when discussing the stability of the beam: jitter where the beam trajectory changes from pulse-to-pulse, emittance degradation due to shifts in the position of the accelerator components, and the degradation of the diagnostic resolution that arises from the beam centroid jitter which can lead to poor convergence of tuning or correction procedures. In the following, we consider the first two issues; the third depends upon the diagnostic design and the operational procedures and is difficult to evaluate without substantial operational experience and/or detailed computer simulation.

There many sources of vibration that can cause the accelerator components to move including the natural seismic motion of the earth as well as man-made cultural noise. The ground motion and vibration can be divided into three regimes: high frequency $(f \gtrsim 10 \mathrm{~Hz})$ where there is little or no spatial correlation of the vibration, the intermediate regime where the lower frequency ground motion tends to be relatively well correlated $(10 \mathrm{~Hz} \gtrsim f \gtrsim 0.1 \mathrm{~Hz})$ and slow uncorrelated motion of components which can be described with the 'ATL' relation [50]; the ATL relation states that after a time $T$, the relative motion of two points, separated by a distance $L$, is equal to $\left\langle\Delta y^{2}\right\rangle=A \star T \star L$.

In general, the effect of the ground vibration must be described with a 2-D power spectrum $P(\omega, k)$ which is a function of both the frequency content as well the spatial correlation of the ground motion [49]. Now, the motion of the beam at a point can be described by

$$
\left\langle\Delta y(t)^{2}\right\rangle=\frac{1}{(2 \pi)^{2}} \int P(\omega, k) G(k) F(\omega) d \omega d k
$$

where $G(k)$ is the spatial response function for the focusing lattice and $F(\omega)$ is the temporal response of the feedback systems. Finally, the growth of the beam spot size can be evaluated in a similar manner except the spatial response function $G$ is now that for the spot size rather than the beam centroid.

Unfortunately, evaluating Eq. (52) usually requires detailed computer calculation. Regardless, one can obtain straight-forward estimates of the effect of the two limiting extremes: uncorrelated high-frequency jitter and the slow ATL motion. The high-frequency motion will cause jitter of the beam centroid while the ATL-drifts primarily result in an increase in the beam emittance since the actual motion of the centroid can be compensated with beam-based feedback systems.

Uncorrelated vibration of the linac quadrupoles $y_{q}$ will deflect the trajectory causing a trajectory motion, at the end of the linac, of

$$
\frac{\left\langle\Delta y^{2}\right\rangle}{\sigma_{y}^{2}} \approx\left\langle y_{q}^{2}\right\rangle \frac{4 \gamma_{i} N_{c e l l} \tan \left(\psi_{c} / 2\right)}{\gamma \epsilon_{y} L_{\text {cell } i}}\left[\left(\frac{\gamma_{f}}{\gamma_{i}}\right)^{1-\alpha}+1\right]
$$

which is expressed in terms of the beam size $\sigma_{y}$.

Similarly, if uncorrected, the ATL motion will cause a trajectory displacement of

$$
\frac{\left\langle\Delta y^{2}\right\rangle}{\sigma_{y}^{2}} \approx 8 A T \frac{N_{c e l l}^{2} \tan \frac{\psi_{c}}{2} \sin \frac{\psi_{c}}{4}}{(3-2 \alpha) \gamma \epsilon_{y}} \frac{(1-\alpha)^{2}}{\left(\gamma_{f}^{1-\alpha}-\gamma_{i}^{1-\alpha}\right)^{2}} \gamma_{f}^{3-2 \alpha}
$$

where the coefficient $A$ depends on the specifics of the site but typically ranges from $A \approx 100 \sim 0.1 \mathrm{~nm} 2 / \mathrm{m}^{2} \mathrm{~s}$ and the factor $\left(\gamma_{f}^{1-\alpha}-\gamma_{i}^{1-\alpha}\right) /(1-\alpha)$ is equal to $\ln \left(\gamma_{f} / \gamma_{i}\right)$ in the limit that $\alpha=1$.

Of course, this trajectory drift could be corrected with beam-based feedback systems at the end of the linac (or portion of the linac if multiple feedback systems are used) but it will still result in emittance degradation. The emittance increase due to the trajectory oscillation is

$$
\langle\Delta \gamma \epsilon\rangle \approx 32 A T \delta_{e}^{2} \frac{N_{c e l l}^{4} \tan \frac{\psi_{c}}{2} \sin \frac{\psi_{c}}{4}}{\cos ^{2} \frac{\psi_{c}}{2}} \frac{1}{(3-2 \alpha)(2-\alpha)^{2}}
$$

where $\delta_{e}$ is the rms deviation from the autophasing energy spread. Typically, $\delta_{e}$ is $20 \sim 30 \%$ of $\sigma_{\text {auto }}$ since the energy variation along the bunch, induced by the rf and longitudinal wakefield, usually does not exactly match the desired form unless the bunch charge distribution is specially shaped. Finally, note that when $\alpha=0$ this expression is similar to that derived in Ref. [51] although the detailed dependence on the phase advance differs slightly. 


\section{DISTRIBUTIONS}

The preceding estimates have been for the 'expected' emittance dilution due to a random distribution of errors. Of course, for any specific set of errors the dilution can deviate from the expected value significantly and thus we need to understand the distribution function for the emittance dilution.

Assuming a Gaussian distribution of errors which cause a single dominant form of dilution, such as accelerator structure misalignments causing emittance dilution due to the transverse wakefields, the dilution will have an exponential distribution [12]:

$$
f(\Delta \gamma \epsilon)=\frac{e^{\frac{-\Delta \gamma \epsilon}{\mu}}}{\mu}
$$

Here, $\mu$ is the expected dilution that could, for example, be calculated from Eq. (41). Strictly, the exponential distribution only arises from a Gaussian distribution of errors but, in a long linac with many errors, the Central Limit Theorem applies and one finds an exponential distribution for the emittance dilution regardless of the detailed distribution function for the errors.

The exponential distribution has a long large amplitude tail, implying that there is a significant probability that any specific set of errors will cause a dilution much larger than the expected dilution. More precisely, with an exponential distribution, there is a $5 \%$ probability that any specific case will exceed three times the expected value. These large dilutions arise when the errors add in phase at the betatron frequency. Fortunately, as will be discussed in the next section, this situation is very easy to measure and correct with slightly more advanced correction techniques.

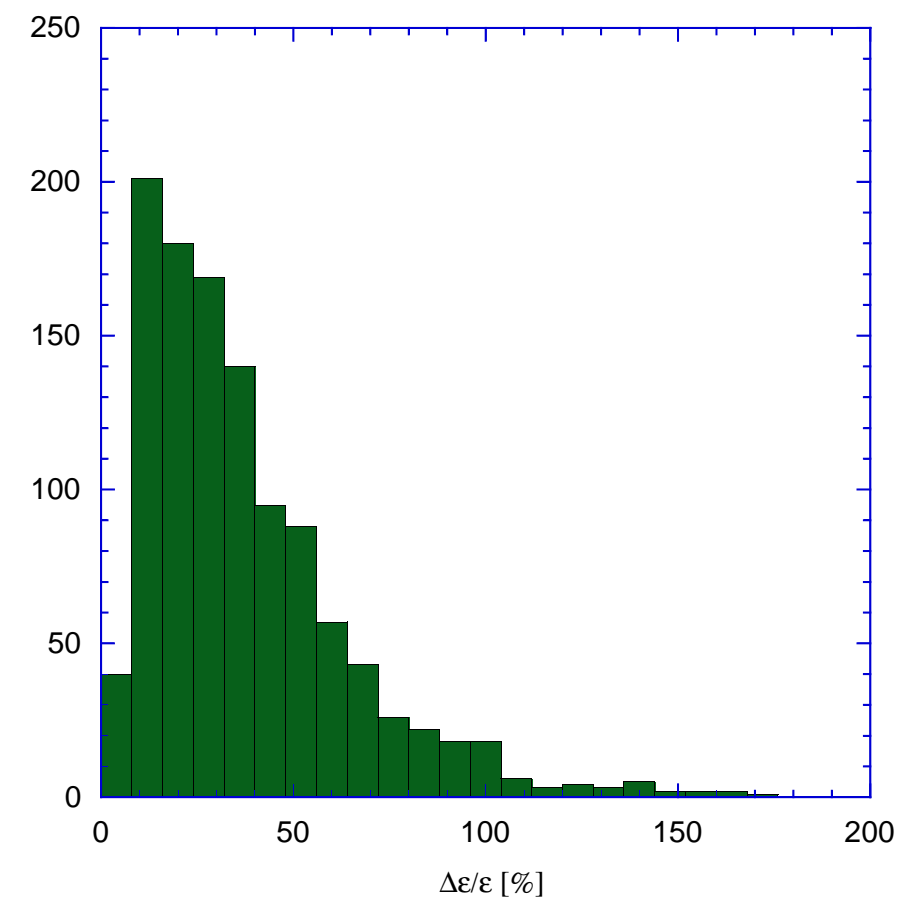

FIG. 9. Histogram of 1100 simulations of accelerator structure misalignments in the NLC linac; the average dilution is $35 \%$ but the distribution of errors is roughly exponential and thus has large tails.

The distribution of dilutions from 1100 different sets of random errors in the NLC linac is plotted in Fig. 9. Here, $10 \mu \mathrm{m}$ random misalignments of the accelerator structures increases, on average, the vertical emittance from $\gamma \epsilon_{y}=$ $3 \times 10^{-8} \mathrm{~m}$-rad to $4.07 \times 10^{-8} \mathrm{~m}$-rad. However, as is evident in the histogram, the distribution is well approximated with an exponential and there is significant possibility that any specific set of errors will have a much larger effect.

Finally, if there are $N$ forms of emittance dilution that are all contributing to the total dilution with equal magnitude, the distribution function for the emittance is described with a $\chi$-squared distribution with $2 N$ degrees-of-freedom. For example, a total emittance dilution of $50 \%$ would have a $\chi$-squared distribution with 4-d.o.f. if it consisted of $25 \%$ dilution due to transverse wakefields and $25 \%$ dilution due to betatron coupling. As the number of degrees-of-freedom increases, the probability of having an emittance much larger than the expected value decreases. Unfortunately, it is difficult to find closed-form solutions for the distribution function in the general case where many forms of dilution 
contribute with different magnitudes but usually there are only one or two dominate effects and the situation can be closely approximated with an exponential ( $\chi$-squared with 2 -d.o.f.) or $\chi$-squared with 4 d.o.f. distribution.

\section{ADVANCED CORRECTION TECHNIQUES}

At this time, a number of more advanced techniques have been developed or proposed to reduce the emittance dilution from the residual errors. The most straightforward technique is to use ' $\epsilon$-bumps'. Here, the measured beam emittance is minimized by adjusting some dilution source upstream of the measurement point. For example, one can reduce the dilution due to the transverse wakefields by appropriately misaligning a few accelerator structures. Such techniques are referred to as 'non-local' correction because the sources of dilution are not reduced, but instead, additional dilutions are added to cancel the effect of the others.

The $\epsilon$-bump technique is routinely used at the Stanford Linear Collider (SLC) to reduce the emittance dilutions by substantial factors, as much as an order of magnitude [38]. Some of the difficulties with the non-local correction techniques are: (1) the beam emittance and tails need to be measured accurately to minimize the dilution, (2) multiple measurement stations may be needed to prevent the dilutions from filamenting, and (3) because the technique cancels the errors by adding another error, it is sensitive to small changes in betatron phase advance that can arise from changes in the quadrupole strengths or energy profile; this results in large fluctuations in the beam emittance at the SLC over a time scale of hours.

Other 'local' correction techniques usually try to measure and correct the emittance dilution sources locally. Typically, this is done by measuring the difference of two, or more, trajectories while some parameter is varied. For example, to align the quadrupoles, one can measure the trajectory while changing the quadrupole power supplies [52-56] or, to measure the accelerator structure misalignments, one can vary the bunch charge or length [35,57].

In all cases, the best alignment that can be attained is limited by the BPM or diagnostic precision and is roughly independent of the magnitude of the misalignments. Although it is difficult to make simple estimates for the effectiveness of these techniques, there are analytic approaches that can be used [58,51]. Regardless, the best method of evaluation (other than experiment) is detailed computer simulation which include many of the systematic errors that exist in a real accelerator. This is important because the techniques frequently rely on measuring small differences in the beam trajectory and can be very sensitive to mundane effects like beam jitter or power supply fluctuations. Finally, one of the other difficulties with these approaches is that the beam emittance is not actually measured and, instead, the dilution sources are inferred from other measurements - thus, it is desirable to include emittance diagnostic stations and use non-local correction to remove any residual dilutions.

\section{SUMMARY}

In this paper, we have described a number of analytic expressions to estimate: the focusing structure in a long linear accelerator, (2) the wakefields and the longitudinal phase space, and (3) the emittance dilutions and jitter sensitivity in a high energy linac. Such expressions provide a valuable tool for performing quick guides to design and performance. In addition, the expressions can be used to highlight the parameter dependence of the various terms and understand the results of more complex computer simulations.

\section{ACKNOWLEDGMENTS}

Numerous people have contributed to this work over the years. In particular, I would like to thank Chris Adolphsen, Ralph Assmann, Reinhard Brinkmann, Jean-Pierre Delahaye, Norbert Holtkamp, Ron Ruth, and Daniel Schulte for entertaining and useful discussions during stays at CERN and DESY and while at SLAC. This work was performed under Department of Energy contract DE-AC03-76SF00515.

[1] J. Arthur et al., "Linac coherent light source (LCLS) design study report," SLAC-R-0521.

[2] See Chapter 5 of Ref. [7]. 
[3] Zeroth-Order Design Report for the Next Linear Collider, T.O. Raubenheimer ed., SLAC-Report-474 (1996).

[4] JLC Design Study, N. Toge ed., KEK Report 97-1 (1997).

[5] T. Shintake et al., "Results from hardware R\&D on C-band RF-system for e+ e- linear collider," Proc. of the XIX Int. Linac Conf., Chicago, IL (1998).

[6] A 3 TeV e+/e- Linear Collider Based on CLIC Technology, G. Guignard, ed., CERN 2000-008 (2000).

[7] Conceptual Design of a $500 \mathrm{GeV}$ e+/e- Linear Collider with Integrated X-ray Facility, R. Brinkmann ed., DESY 97-48 (1997).

[8] T. H. Fieguth et al., "Injection system for the PEP-II asymmetric B factory at SLAC," Presented at 3rd Eur. Part. Acc. Conf., Berlin, Germany (1992) p. 1443.

[9] A. Enomoto, S. Anami, T. Kamitani, H. Tetsuo, T. Shidara and I. Sato, "Linac upgrade plan for the KEK B-factory," Submitted to 1993 Part. Acc. Conf., Washington, DC (1993) p. 590.

[10] R.D. Ruth, "Emittance Preservation in Linear Colliders," Proc. of the 1986 CERN/US Part. Acc. School, South Padre Is., TX (1986) p. 440.

[11] H. Henke, "Transport and Acceleration of Low-Emittance Electron Beams," Proc. of the 1988 Linear Acc. Conf., Williamsburg, VA (1988).

[12] T.O. Raubenheimer, "The Generation and Acceleration of Low Emittance Flat Beams for Future Linear Colliders," Ph.D. Thesis, Stanford University, Stanford CA (1991).

[13] G. Guignard, J. Hagel, "Theory of Single Bunch Stability and Dynamics in Linacs with Strong Wakefields and Misalignments," Nucl. Instr. Meth., A434: 179 (1999).

[14] J. Seeman, "The Stanford Linear Collider," Ann. Rev. Nucl. Part. Sci., 41, 389 (1990).

[15] F. Zimmermann, T.O. Raubenheimer, "Longitudinal Space Charge in Final Focus Systems for Linear Colliders," Nucl. Instr. Meth., A390: 279 (1997).

[16] L. Merminga, et. at., "Transverse phase space in the presence of dispersion," Proc. of 1991 IEEE Part. Acc. Conf., San Francisco, CA (1991) p. 461.

[17] G. Guignard, "Lattice Scaling and Emittance Control in the CLIC Main Linac," CLIC-Note-193 (1993).

[18] S. Heifets, S. Kheifets, "High Frequency Limit of the Longitudinal Coupling Impedance," Phys. Rev., D39: 960 (1989).

[19] See for example articles in: Proc. of the Work. on Impedance Beyond Cutoff, Part. Acc., 25: 61 (1990).

[20] A. Novokhatski, A. Mosnier, "Short Bunch Wake Potentials for a Chain of TESLA Cavities," DAPNIA/SEA-9608 (1996).

[21] R.B. Palmer, "Prospects for High Energy $e^{+} e^{-}$Linear Colliders," Ann. Rev. Nucl. Part. Sci., 40: 529 (1990).

[22] P. Wilson, AIP Conf. Proc. 87, Amer. Inst. Phy. (1982) p. 450.

[23] K. Yokoya, private communication (1997).

[24] K. Bane, A. Mosnier, A. Novokhatskii, K. Yokoya, "Calculations of the Short Range Longitudinal Wakefields in the NLC Linac," Proc. of 1998 Eur. Part. Acc. Conf., Stockholm, Sweden (1998).

[25] G.A. Loew, J.M. Wang, "Minimizing the Energy Spread within a Single Bunch by Shaping its Charge Distribution," Proc. of 1985 IEEE Part. Acc. Conf., Vancouver, B.C., Canada (1985) p. 3228.

[26] F.J. Decker, R. Holtzapple, T.O. Raubenheimer, "Over Compression, a Method to Shape the Longitudinal Bunch Distribution for a Reduced Energy Spread," Proc. of 17th Int. Linear Acc. Conf., Tsukuba, Japan (1994) p. 47.

[27] For example see: K.L. Bane, T.O. Raubenheimer, J.T. Seeman, "Electron Transport of a Linac Coherent Light Source (LCLS) using the SLAC Linac," Proc. of 1993 IEEE Part. Acc. Conf., Washington D.C. (1993) p. 3933.

[28] A. Chao, B. Ritcher, C.Y. Yao, "Beam Emittance Growth Caused by Transverse Deflecting Fields in a Linear Accelerator," Nucl. Instr. Meth., 178: 1 (1980).

[29] V. Balakin, A. Novokhatski, V. Smirnov, "Longitudinal Dynamics in the VLEPP Collider," Proc. 12th Int. High Energy Accel., Batavia, IL, (1983) p. 119.

[30] W. Schnell, "Microwave Quadrupoles for Linear Colliders," CLIC Note-34 (1987).

[31] D. Chernin, A. Mondelli, "Effect of Energy Spread on the Single-Bunch Dipole Beam Break-Up Instability in a High-Energy Linac," Part. Acc., 24: 177 (1989).

[32] K. Yokoya, "Cumulative Beam Break-Up in Large-Scale Linacs," DESY 86-084 (1986).

[33] G. Stupakov, "BNS Damping of Beam Breakup Instability," SLAC-AP-108 (1997).

[34] V. Balakin, Proc. 1988 Workshop on Linear Colliders, SLAC-report-355, (1989) p. 55.

[35] T.O. Raubenheimer, K. Kubo, "A Technique of Measuring and Correcting Emittance Dilutions due to Accelerator Structure Misalignments," Nucl. Inst. Meth., A370: 303 (1996).

[36] C. Adolphsen, K. Bane, J.T. Seeman, "Effect of Wakefields on First-Order Transport in the SLC Linac," Proc. of 1991 IEEE Part. Acc. Conf., San Francisco, CA (1991) p. 3207.

[37] For a discussion see: T.O. Raubenheimer, "The Preservation of Low Emittance Flat Beams," Proc. of 1993 IEEE Part. Acc. Conf., Washington D.C. (1993) p. 11.

[38] J.T. Seeman, F.J. Decker, I. Hsu, "The Introduction of Trajectory Oscillations to Reduce Emittance Growth in the SLC Linac," Proc. of 15th Int. Conf. High Energy Acc., Hamburg, Germany (1992) p. 879.

[39] M. Syphers, "Injection Mismatch and Phase Space Dilution," FN-458 (1987).

[40] F.J. Decker, et. at., "Dispersion and Betatron Matching into the Linac," Proc. of the 1991 Part. Acc./ Conf., San Francisco, CA (1991) p. 6. 
[41] M. Syphers, T. Sen, D. Edwards, "Amplitude Function Mismatch," Proc. of 1993 IEEE Part. Acc. Conf., Washington D.C. (1993) p. 134.

[42] T.O. Raubenheimer, F.-J. Decker, J.T. Seeman, "Beam Distribution Function after Filamentation," Proc. 1995 Part. Acc. Conf., Dallas, TX (1995) p. 3291.

[43] R. Helm, G.A. Loew, "Beam Break-up," Linear Accelerators, eds: Lapostolle and Septier, North Holland Books, Amster$\operatorname{dam}(1970)$.

[44] R.L. Gluckstern, R.K. Cooper, P.J. Channell, "Cumulative Beam Break-Up in RF Linacs," Part. Acc., 16: 125 (1985).

[45] K. Thompson, R. Ruth, "Controlling Transverse Multibunch Instabilities in Linacs of High-energy Linear Colliders," Phys. Rev. D, 41: 964 (1990).

[46] K. Bane, C. Adolphsen, K. Kubo, K. Thompson, "Issues in Multi-Bunch Emittance Preservation in the NLC," Proc. of the 4th Europ. Part. Conf., London, England (1994) p. 1114.

[47] G.V. Stupakov, "Suppression of beam breakup instability in a long train by introducing energy spread between the bunches," physics/0008008.

[48] C. L. Bohn and K. Y. Ng, "Preserving high multibunch luminosity in linear colliders," Phys. Rev. Lett., 85, 984 (2000).

[49] For example see: A. Sery, O. Napoly, "Influence of Ground Motion on the Time Evolution of Beams in Linear Colliders," Phys. Rev. E, 53: 5323 (1996).

[50] V. Shiltsev, "Space-Time Ground Diffusion: the ATL Law for Accelerators," Proc. 4th Int. Work. on Acc. Align., Tsukuba, Japan (1995).

[51] A. Sery, A. Mosnier, "Spectral Analysis of Alignment Techniques for Linear Colliders," Phys. Rev., E56: 3558, (1997).

[52] One of the standard approaches to determine the beam offset in a quadrupole is to vary the quadrupole strength and measure the resulting deflection.

[53] C. Adolphsen, et al., "Beam Based Alignment Technique for the SLC Linac," Proc. of the 1989 IEEE Part. Acc. Conf., Chicago, IL (1989) p. 977.

[54] T.O. Raubenheimer, R.D. Ruth, "A Dispersion-Free Trajectory Correction Technique for Linear Colliders," Nucl. Instr. Meth., A302: 191 (1991).

[55] T.O. Raubenheimer, "A New Technique of Correcting Emittance Dilutions in Linear Colliders," Nucl. Instr. Meth., A306: 61 (1991).

[56] R. Assmann, T. Chen, F.J. Decker, M. Minty, T.O. Raubenheimer, R. Siemann, "Quadrupole Alignment and Trajectory Correction for Future Linear Colliders: SLC Tests of a Dispersion-Free Steering Algorithm," Proc. of the Fourth Int. Work. on Acc. Alignment, KEK Proceedings 95-12, Tsukuba, Japan (1995).

[57] C. Fischer, "Improved CLIC Performance using the Beam Response for Correcting Alignment Errors," Proc. 1995 IEEE Part. Acc. Conf., Dallas, TX (1995) p. 731.

[58] C. Adolphsen, T.O. Raubenheimer, "Method to Evaluate Steering and Alignment Algorithms for Controlling Emittance Growth," Proc. of 1993 IEEE Part. Acc. Conf., Washington D.C. (1993) p. 417. 